\title{
Poster Number 16153 \\ Preliminary results of human soft tissues adhesion around different treated healing abutments
}

\author{
Ghinassi Barbara $D D S, P H D_{2}$, \\ D'Addazio Gianmaria $D D S$, $P H D$, Murmura Giovanna DDS, \\ Piattelli Maurizio MD, DDS, Sinjari Bruna DDS, PHD.
}

BASIC RESEARCH

1 Department of Medical, Oral and Biotechnological Sciences, University "G. d'Annunzio" Chieti-Pescara, Chieti, Italy;

2 Department of Medicine and Aging Sciences, University “G. d'Annunzio” Chieti-Pescara, Chieti, Italy;

\section{Background and Aim}

Long-term implant success has been shown to be related to the presence of a soft tissue barrier, which prevents bacterial penetration. The quality of this mucosal attachment is influenced by the properties of the implant components that are placed in contact with the soft tissue. Although, different study has been performed there is still lack of information on which is the implant surface that better promote the soft tissue cells adhesion. The aim of the present study is to evaluate the adhesion of human soft tissues surrounding a custom-made healing abutments.

\section{Methods and Materials}

A total of 21 healing abutments with alternate laser-conditioned and machined surfaces with attached the gingival biopsies have been collected after 4 weeks of healing. The "G.d'Annunzio" Chieti-Pescara University Ethic Committee has approved this clinical trial $\mathrm{N}^{\circ} 22$ of 18.10.2018. Paraffin sections of human gingiva surrounding the healing abutments were prepared from 10 subjects. Hematossilin Eosin and Tricrome staining and image analysis were performed to measure and compare the morphological differences among junctional epithelium, sulcular epithelium and the subepithelial connective tissue of the gingiva touching the smooth and the laser-conditioned surface of the healing abutment. Maygrunwald Giemsa staining and immunofluorescence for CD63 were performed to identify inflammation area.

\section{Results}

The region of the gingiva adherent to the laser-conditioned showed a regular morphology in all its parts (junctional epithelium, sulcular epithelium and subepithelial connective tissue). By contrast, the region of the gingiva adherent to the machined surfaces showed several stress hallmarks:

a) disruption and discontinuities in the basal membrane associated with areas of increased inflammation evident both in the epithelium and, in a larger extent, in the subepithelial connective (abundant presence of CD63 positive area that is surface marker specific for neuthrophils present in inflamed tissue and it is not expressed by neutrophils present in healthy tissue).

b) The correct keratinization process is defective or absent since the corneum status present still nucleated cells or a very thin enucleated cells layer compared to the regular corneal layer of the epithelium of the part of gingiva facing the laser-conditioned surface

\section{Conclusion}
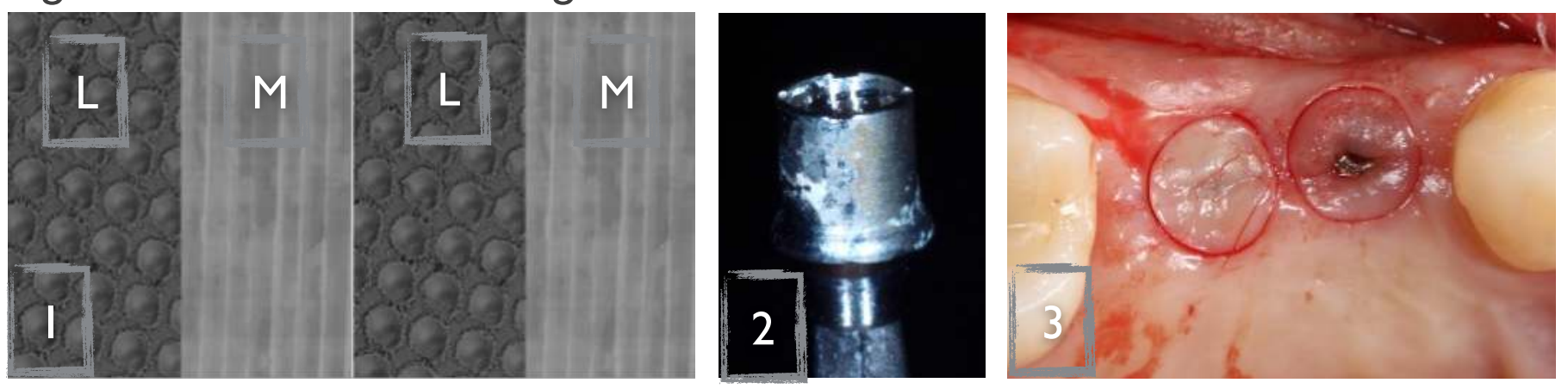

Img.1: vertical 2D representation of tested he
alternately (L= laser-conditioned; $M=$ machined)

Img.2: Clinical image of healing abutment.

Img.3: Clinical image of surgical biopsy performed around the tested healing abutment. A circular punch of soft tissue was performed.
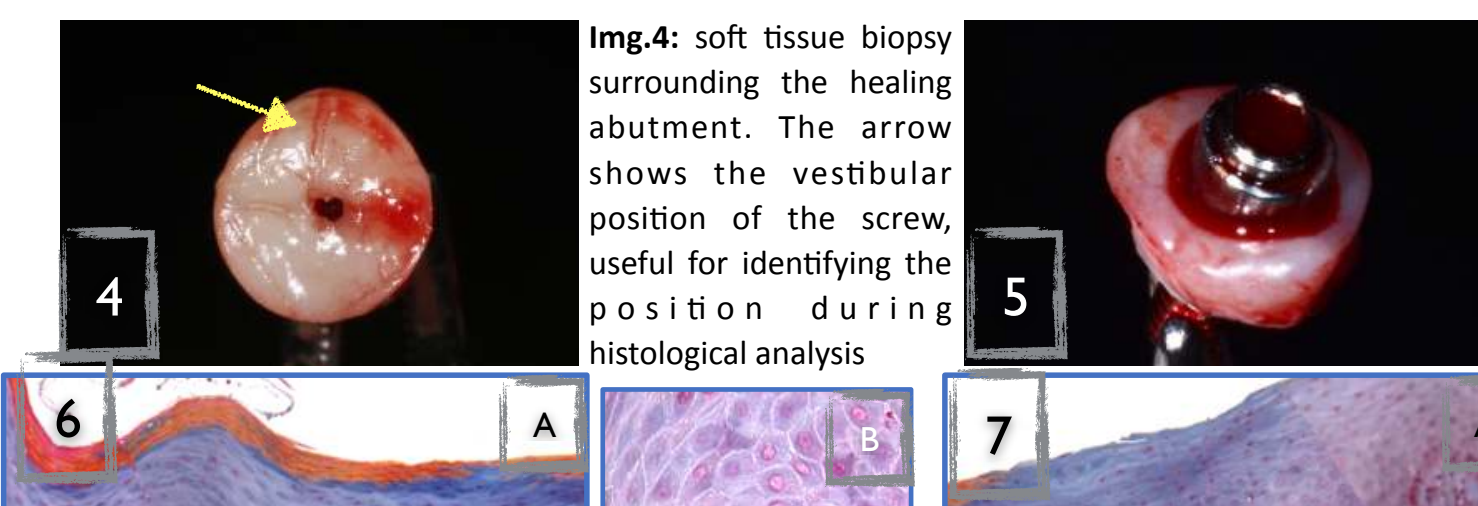

Img.5: other tissue biopsy with different soft tissue height.

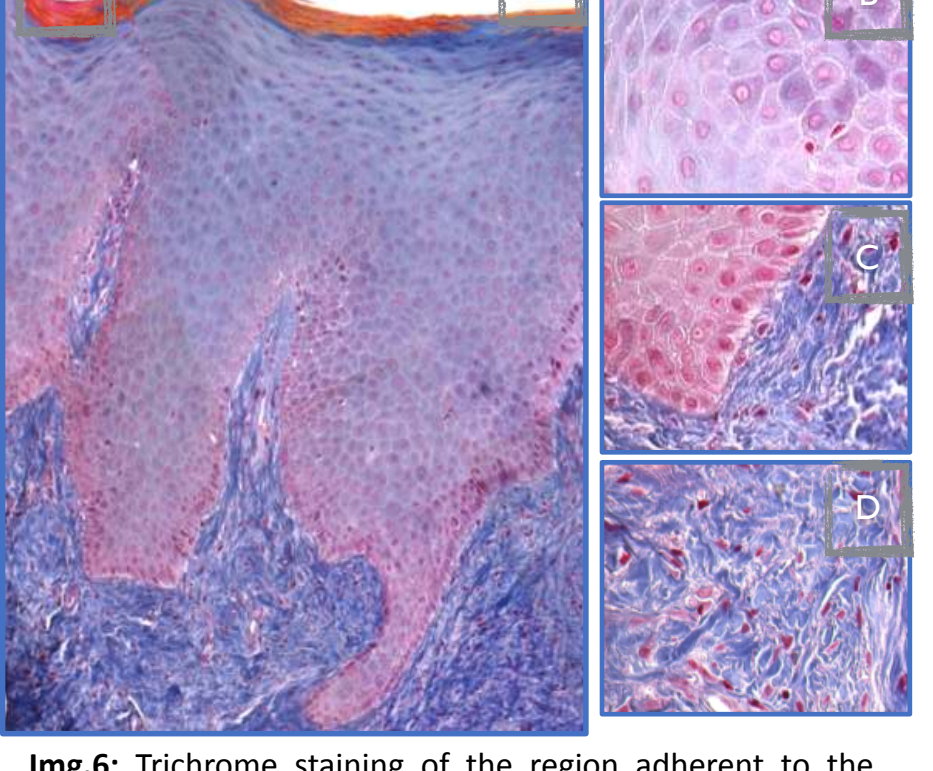

Img.6: Trichrome staining of the region adherent to the laser-conditioned surface evidences regular morphology of the gingiva ([A] Magnification 10x), showing physiological keratinization as well as normal epithelial layers, basal lamina and connective tissue stroma ([B], [C], [D] resprtively, magnification 40x).
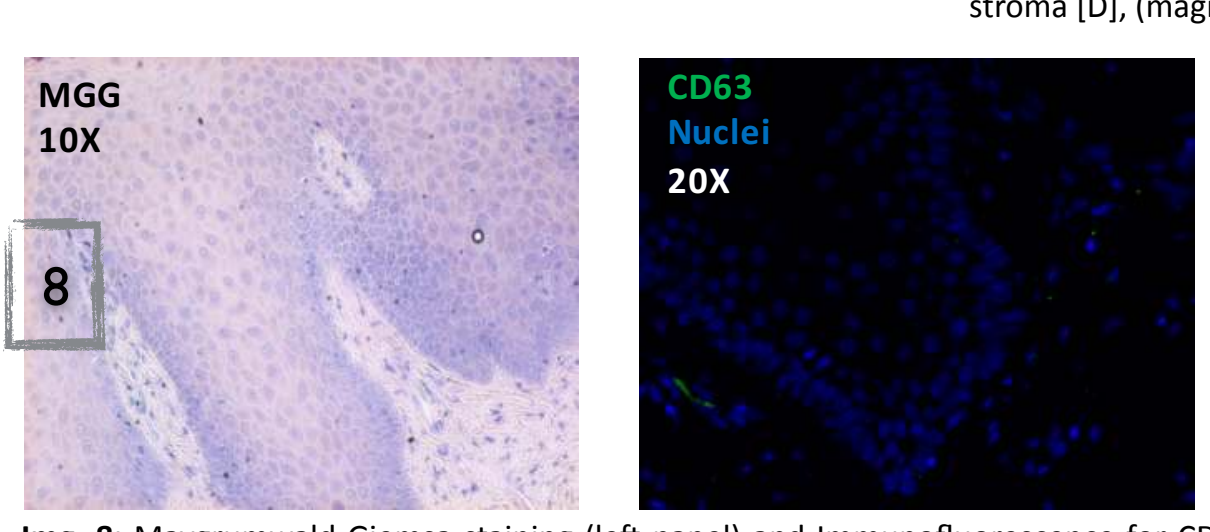
Img.7: Trichrome staining of the region adherent to the machined surface evidences irregularities at the epithelium/stroma surface and the total absence of keratinized external layer ([A] Magnification 10x). The continuity of the basal lamina of the epithelium is disrupted [C] and associated with area of increased imflammation both in the epithelium [B] and
stroma [D], (magnification 40x).

These data suggest that the use of laser treated surface on healing abutment could positively influence the healing. A better distribution and cell morphology on test surface could encourage the clinical use of this treatment. The laser treated surface seems to influence the cell adhesion and the inflammatory infiltrate of the soft tissue. However, further study will be necessary to better understand biological aspects related to soft tissue adhesion on abutment and prosthetic materials

References

Img. 8: Maygrumwald Giemsa staining (left panel) and Immunofluorescence for CD63, a marker for neutrophil granule release absence of flogistic area

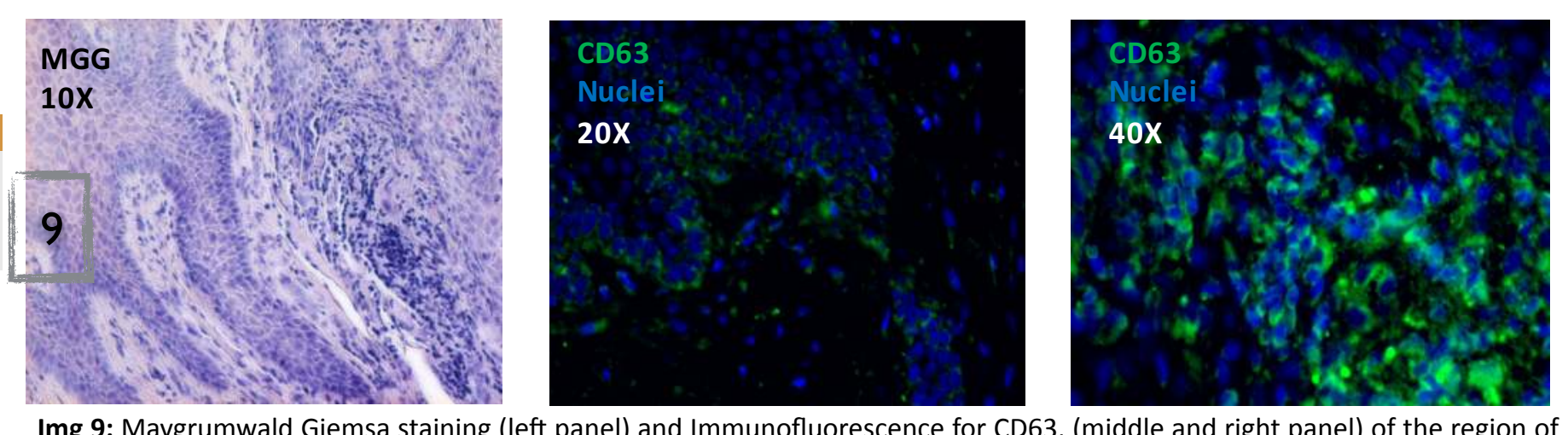

Img 9: Maygrumwald Giemsa staining (left panel) and Immunofluorescence for CD63, (middle and right panel) of the region of the gingiva adherent to the machined surface
maily located in the connective tissue stroma 